\title{
Hyperhomocysteinemia Is Associated With Lipid Profiles and Lipid Ratio in Patients With Coronary Artery Disease
}

\section{Dong-Feng Wu}

The People's Hospital of Guangxi Zhuang Autonomous Region

Qin-Chen Liao

The People's Hospital of Guangxi Zhuang Autonomous Region

Feng Lu

The People's Hospital of Guangxi Zhuang Autonomous Region

Zhou Wang

The People's Hospital of Guangxi Zhuang Autonomous Region

Kun Yu

The People's Hospital of Guangxi Zhuang Autonomous Region

Jin-Long Deng ( $\nabla$ djl_gx@163.com )

The People's Hospital of Guangxi Zhuang Autonomous Region

\section{Research Article}

Keywords: Coronary artery disease, Hyperhomocysteinemia, Serum lipid level, Lipid ratios

Posted Date: September 17th, 2021

DOI: https://doi.org/10.21203/rs.3.rs-877601/v1

License: (1) This work is licensed under a Creative Commons Attribution 4.0 International License. Read Full License 


\section{Abstract}

Objective: This study aimed to investigate the correlation of Hyperhomocysteinemia (HHcy) or serum homocysteine (Hcy) levels with lipid levels and lipid ratios in individuals with coronary artery disease(CAD).

Methods: A total of 1646 subjects with suspected CAD were divided into CAD or control groups. Serum Hcy, total cholesterol(TC), triglycerides(TGs), high-density lipoprotein cholesterol(HDL-C), low-density lipoprotein cholesterol(LDL-C), apolipoprotein(Apo)AI and ApoB concentrations were detected.

Results: Serum TC, LDL-C and ApoB in control subjects with HHcy were lower than those in individuals with normal Hcy, and serum HDL-C and ApoAl in CAD subjects with HHcy were lower than those in individuals with normal $\mathrm{Hcy}(P<0.05)$. The correlation analysis showed that serum TGs, LDL-C, ApoAl and $\mathrm{HDL}-\mathrm{C}$ were correlated with $\mathrm{Hcy}(P<0.05)$. There are different $\mathrm{HHcy}$ trends affecting the ratios of $\mathrm{TC} / \mathrm{HDL}-\mathrm{C}$ and LDL/HDL-C between the CAD and controls $\left(P_{\text {interaction }}\right.$ for TC/HDL-C $=0.025 ; P_{\text {interaction }}$ for $\mathrm{LDL} / \mathrm{HDL}-\mathrm{C}=0.033)$. CAD patients with $\mathrm{HHcy}$ had a higher ratio of TC/HDL-C $(P=0.022)$ and LDL/HDL$\mathrm{C}(P=0.045)$ than those with normal Hcy, but in the controls, the subjects with HHcy exhibited a trend toward a decreased ratio of TC/HDL-C $(P=0.481)$ and LDL/HDL-C $(P=0.303)$.

Conclusion: HHcy was related to the atherogenic lipid profile in patients with CAD. The lipid ratio is more suitable for assessing the effect of HHcy on CAD.

\section{Introduction}

Coronary artery disease (CAD) is the leading cause of death worldwide and remains a major health problem in both developed and developing countries ${ }^{[1]}$. Traditional lipid parameters, such as increased serum levels of total cholesterol (TC), triglycerides (TGs), low-density lipoprotein cholesterol (LDL-C), and apolipoprotein (Apo) B, or low levels of high-density lipoprotein cholesterol (HDL-C) and ApoAl, are among the most important modifiable risk factors for $\mathrm{CAD}^{[2,3]}$. It has also been reported that nontraditional lipid profiles (lipid ratio) are a powerful predictor for cardiovascular disease (CVD) ${ }^{[4-13]}$. For instance, after a 10-year followed up of a prospective cohort study including 15,632 initially healthy US women, it was suggested that the TC/HDL-C ratio was as good as or better than apolipoprotein fractions for the prediction of future cardiovascular events ${ }^{[7]}$. TG/HDL-C is a highly atherogenic marker of insulin resistance and cardiometabolic risk and correlates positively with LDL phenotype B and LDL particle concentration and inversely with small, dense LDL particle size ${ }^{[9]}$. Moreover, TC/HDL-C and LDL-C/HDL-C have been found to be independent indicators of vascular risk with greater predictive value than isolated lipid levels ${ }^{[6-8,12]}$.

Despite therapeutic advances that control many risk factors, such as statins that have decreased LDL-C levels to lower levels than previously possible, CVD remains a major cause of morbidity and mortality worldwide ${ }^{[14]}$. Hyperhomocysteinemia (HHcy) has been regarded as a new risk factor for CVD. Folate 
deficiency and the methylene tetrahydrofolate reductase (MTHFR) C677T polymorphism are considered the main causes of HHcy. In China, 1/4 of the population has the MTHFR C677T TT genotype, and due to dietary habits and the high prevalence of smoking and alcohol consumption, it is easy to lack folic acid, vitamin $\mathrm{B} 6$ and $\mathrm{B} 12$, thus resulting in $\mathrm{HHcy}$ in our population ${ }^{[15]}$. HHcy acts through various mechanisms, including vascular endothelium damage, stimulation of smooth muscle cell proliferation, and thrombosis activation ${ }^{[16,17]}$. Previous studies have also established possible links among HHcy, dyslipidemia, and atherosclerosis ${ }^{[18-19]}$. Several studies relating HHcy to disturbed HDL-C metabolism have shown that Hcy can reduce circulating HDL-C by inhibiting ApoAl protein synthesis and enhancing HDL-C clearance [21-24].

In the present study, a negative correlation between HHcy and HDL-C and ApoAl was observed. However, perhaps due to the effects of lipid-lowering drugs, HHcy was also negatively associated with serum TC, TGs and ApoB. Therefore, it is difficult to determine whether HHcy promotes atherosclerosis or protects against atherosclerosis by evaluating traditional lipid parameters. Thus, this study aims to further investigate the association of HHcy with lipid ratio in CAD patients.

\section{Materials And Methods}

\section{Objects}

A total of 1646 patients with suspected CAD were recruited from a population of hospitalized patients in The People's Hospital of Guangxi Zhuang Autonomous Region. Coronary angiography (CAG) was performed in all enrolled subjects. Such as described in detail in our previous study ${ }^{[25]}$, CAD was defined as significant coronary stenosis ( $\geq 50 \%$ ) in at least one of the three main coronary arteries or their major branches (branch diameter $\geq 2 \mathrm{~mm}$ ). Subjects with congenital heart disease, cardiomyopathy, valvular disease, autoimmune disease, or type I diabetes mellitus were excluded. The study protocol was approved by the Ethics Committee of The People's Hospital of Guangxi Zhuang Autonomous Region. Informed consent was obtained from all subjects after they received a full explanation of the study.

\section{Blood sample collection and laboratory methods}

After an overnight fast of at least $12 \mathrm{~h}$, a venous blood sample was obtained from the forearm of each patient, which were described in detail in our previous study ${ }^{[26]}$, serum Hcy was measured using an enzymatic cycling method. Serum TC, LDL-C, HDL-C, and TGs were measured by enzymatic methods with commercially available kits on a Beckman Coulter Automatic Analyzer. Serum ApoAl and ApoB levels were detected by immunoturbidimetric immunoassay. All of these biochemical analyses were performed at the Clinical Laboratory of The People's Hospital of Guangxi Zhuang Autonomous Region. According to results from previous studies ${ }^{[26]}$, $\mathrm{Hcy} \geq 15 \mu \mathrm{mol} / \mathrm{L}$ is often defined as HHcy.

\section{Statistical analyses}


The statistical software package SPSS 21.0 (SPSS Inc., Chicago, Illinois) was used for the statistical analyses. Quantitative variables are expressed as the mean \pm standard deviation. Qualitative variables are expressed as raw counts and percentages. The differences in the general characteristics between patients and controls were tested by Student's unpaired t-test or chi-square analysis. Comparison of blood lipid parameters between different groups was performed by analysis of covariance (ANCOVA). Multivariate linear regression analysis with stepwise modeling was performed to evaluate the association of serum Hcy levels with serum lipid levels. Sex, age, and BMI were adjusted for the statistical analysis. A two-tailed $P$ value of less than 0.05 was considered statistically significant.

\section{Results}

\section{General characteristics and serum lipid levels}

Table 1 shows the general characteristics and serum lipid parameters of the study population. Compared with the controls, the CAD patients had a higher proportion of males and individuals with hypertension, lower levels of HDL-C and ApoAl, and a higher level of serum Hcy ( $P$ for all $<0.05)$. Maybe due to the effects of lipid-lowering drugs, there were no significant differences in the levels of serum TC, TGs, LDL-C, or $A p o B$; there were also no significant differences in the age distribution or the prevalence of diabetes between the two groups $(P>0.05$ for all). 
Table 1

The general characteristics and serum homocysteine level in CAD and controls

\begin{tabular}{|lllll|}
\hline Parameters & Controls & CAD & $t / x^{2}$ & $P$ \\
\hline $\mathrm{N}$ & 774 & 872 & - & - \\
\hline Age & $63.66 \pm 29.81$ & $63.27 \pm 10.40$ & 0.362 & 0.730 \\
\hline Male/Female & $432 / 342$ & $634 / 238$ & 51.273 & 0.000 \\
\hline Hypertension & $198(25.6)$ & $269(30.8)$ & 5.598 & 0.018 \\
\hline Diabetes & $88(11.4)$ & $106(12.2)$ & 0.244 & 0.621 \\
\hline TC (mmol/L) & $4.53 \pm 1.13$ & $4.44 \pm 1.22$ & 1.654 & 0.097 \\
\hline TG (mmol/L) & $1.56 \pm 0.95$ & $1.66 \pm 1.12$ & -1.940 & 0.053 \\
\hline HDL-C (mmol/L) & $1.15 \pm 0.30$ & $1.11 \pm 0.32$ & 2.467 & 0.014 \\
\hline LDL-C (mmol/L) & $2.74 \pm 0.80$ & $2.66 \pm 0.98$ & 1.810 & 0.071 \\
\hline ApoAl (g/L) & $1.18 \pm 0.26$ & $1.07 \pm 0.31$ & 7.350 & 0.000 \\
\hline ApoB (g/L) & $0.91 \pm 0.25$ & $0.90 \pm 0.29$ & 0.277 & 0.780 \\
\hline Hcy $($ umol/l) & $13.50 \pm 5.84$ & $15.11 \pm 5.20$ & -5.928 & 0.000 \\
\hline $\begin{array}{l}\text { TC, total cholesterol; TG, triglyceride; HDL-C, high-density lipoprotein cholesterol; LDL-C, low-density } \\
\text { lipoprotein cholesterol; ApoAl, apolipoprotein Al; ApoB, apolipoprotein B; Hcy: Homocysteine }\end{array}$ \\
\hline
\end{tabular}

\section{Hyperhomocysteinemia and serum lipid levels}

As shown in Table 2, when statistical analysis was performed between the normal Hcy and HHcy groups according to whether the serum homocysteine concentration was greater than $15 \mu \mathrm{mol} / \mathrm{L}$, the levels of serum TC, LDL-C and ApoB in the HHcy group were significantly lower than those in the normal Hcy group; the levels of serum HDL-C and ApoAl in the HHcy group were significantly lower than those in the normal Hcy group ( $P$ for all $<0.05$ ). There were no significant interactions between $\mathrm{HHcy}$ and disease in terms of the serum lipid levels $(P$ for all $>0.05)$. 
Table 2

$\mathrm{HH}$ cy and serum lipid levels in CAD patients and controls

\begin{tabular}{|c|c|c|c|c|c|c|c|}
\hline Group & $\mathbf{N}$ & $\begin{array}{l}\text { TC } \\
(\mathrm{mmol} / \mathrm{L})\end{array}$ & $\begin{array}{l}\text { TG } \\
(\mathrm{mmol} / \mathrm{L})\end{array}$ & $\begin{array}{l}\text { HDL-C } \\
(\mathrm{mmol} / \mathrm{L})\end{array}$ & $\begin{array}{l}\text { LDL-C } \\
\text { (mmol/L) }\end{array}$ & $\begin{array}{l}\text { ApoAl } \\
(g / L)\end{array}$ & $\begin{array}{l}\text { ApoB } \\
(g / L)\end{array}$ \\
\hline \multicolumn{8}{|l|}{ Control } \\
\hline Normal Hcy & 532 & $\begin{array}{l}4.60 \pm \\
1.20\end{array}$ & $\begin{array}{l}1.61 \pm \\
0.99\end{array}$ & $\begin{array}{l}1.16 \pm \\
0.31\end{array}$ & $\begin{array}{l}2.80 \pm \\
0.83\end{array}$ & $\begin{array}{l}1.19 \pm \\
0.26\end{array}$ & $\begin{array}{l}0.92 \pm \\
0.26\end{array}$ \\
\hline HHcy & 242 & $\begin{array}{l}4.37 \pm \\
0.94\end{array}$ & $\begin{array}{l}1.46 \pm \\
0.83\end{array}$ & $\begin{array}{l}1.13 \pm \\
0.27\end{array}$ & $\begin{array}{l}2.61 \pm \\
0.66\end{array}$ & $\begin{array}{l}1.16 \pm \\
0.24\end{array}$ & $\begin{array}{l}0.88 \pm \\
0.21\end{array}$ \\
\hline$F$ & & 6.856 & 3.677 & 1.827 & 9.039 & 2.119 & 5.013 \\
\hline$P$ & & 0.009 & 0.056 & 0.177 & 0.003 & 0.146 & 0.025 \\
\hline \multicolumn{8}{|l|}{ CAD } \\
\hline Normal Hcy & 484 & $\begin{array}{l}4.49 \pm \\
1.32\end{array}$ & $\begin{array}{l}1.69 \pm \\
1.20\end{array}$ & $\begin{array}{l}1.15 \pm \\
0.30\end{array}$ & $\begin{array}{l}2.70 \pm \\
1.05\end{array}$ & $\begin{array}{l}1.10 \pm \\
0.25\end{array}$ & $\begin{array}{l}0.91 \pm \\
0.31\end{array}$ \\
\hline HHcy & 388 & $\begin{array}{l}4.37 \pm \\
1.06\end{array}$ & $\begin{array}{l}1.63 \pm \\
1.01\end{array}$ & $\begin{array}{l}1.07 \pm \\
0.34\end{array}$ & $\begin{array}{l}2.62 \pm \\
0.86\end{array}$ & $\begin{array}{l}1.04 \pm \\
0.37\end{array}$ & $\begin{array}{l}0.89 \pm \\
0.25\end{array}$ \\
\hline$F$ & & 1.783 & 0.525 & 10.202 & 1.279 & 6.197 & 0.650 \\
\hline$P$ & & 0.182 & 0.469 & 0.001 & 0.258 & 0.013 & 0.420 \\
\hline $\begin{array}{l}P \\
\text { interaction }\end{array}$ & & 0.303 & 0.615 & 0.382 & 0.240 & 0.667 & 0.363 \\
\hline
\end{tabular}

\section{HHcy and nontraditional lipid profiles (lipid ratios)}

As shown in Table 3, there are different trends in HHcy affecting the ratio of TC/HDL-C and LDL-C/HDL-C between the CAD patients and controls $\left(P_{\text {interaction }}\right.$ for TC/HDL-C $=0.025$; $P_{\text {interaction }}$ for LDL-C/HDL-C $=$ 0.033). CAD patients with HHcy had a significantly higher ratio of TC/HDL-C than those with normal Hcy (normal Hcy: $4.10 \pm 1.32$ vs HHcy: $4.43 \pm 2.60, P=0.025$ ), but in the controls, the subjects with HHcy exhibited a trend toward a decreased ratio of TC/HDL-C (normal Hcy: 4.11 \pm 1.03 vs HHcy: $4.05 \pm 1.11, P=$ 0.481). CAD patients with HHcy had a higher ratio of LDL/HDL-C (normal Hcy: $2.48 \pm 1.07$ vs HHcy: 2.65 $\pm 1.36 ; P=0.045)$, but in the controls, the subjects with HHcy exhibited a trend toward a decreased ratio of LDL/HDL-C (normal Hcy: $2.52 \pm 0.80$ vs HHcy: $2.45 \pm 0.85 ; P=0.303$ ). 
Table 3

Lipid ratios in the HHcy and normal Hcy groups

\begin{tabular}{|llllll|}
\hline Group & $\mathrm{N}$ & $\mathrm{TC} / \mathrm{HDL}-\mathrm{C}$ & $\mathrm{TG} / \mathrm{HDL}-\mathrm{C}$ & LDL/HDL-C & ApoB/ApoAl \\
\hline Control & & & & \\
\hline Normal Hcy & 532 & $4.11 \pm 1.03$ & $1.50 \pm 1.02$ & $2.52 \pm 0.80$ & $0.80 \pm 0.27$ \\
\hline HHcy & 242 & $4.05 \pm 1.11$ & $1.43 \pm 1.00$ & $2.45 \pm 0.85$ & $0.79 \pm 0.27$ \\
\hline$F$ & & 0.498 & 0.781 & 1.063 & 0.210 \\
\hline$P$ & & 0.481 & 0.377 & 0.303 & 0.647 \\
\hline CAD & & & & \\
\hline Normal Hcy & 484 & $4.10 \pm 1.32$ & $1.60 \pm 1.27$ & $2.48 \pm 1.07$ & $0.87 \pm 0.35$ \\
\hline HHcy & 388 & $4.43 \pm 2.60$ & $1.79 \pm 2.18$ & $2.65 \pm 1.36$ & $0.92 \pm 0.36$ \\
\hline$F$ & 5.276 & 2.431 & 3.860 & 4.372 \\
\hline$P$ & 0.022 & 0.119 & 0.045 & 0.037 \\
\hline$P$ interaction & 0.025 & 0.144 & 0.033 & 0.094 \\
\hline $\begin{array}{l}\text { TC, total cholesterol; TG, triglyceride; HDL-C, high-density lipoprotein cholesterol; LDL-C, low-density } \\
\text { lipoprotein cholesterol; ApoAl, apolipoprotein Al; ApoB, apolipoprotein B; HHcy: }\end{array}$ & \\
\hline Hyperhomocysteinemia
\end{tabular}

\section{Correlation of Hcy and serum lipid parameters}

The correlation between the Hcy and serum lipid parameters is shown in Table 4. Sex, age, BMI, diabetes, and hypertension were excluded from the statistical analysis. The serum LDL-C and ApoAI levels were correlated with Hcy in the combined CAD and control populations $(P<0.05)$. The levels of serum HDL-C and TGs in the control group and HDL-C and ApoAl were correlated with Hcy $(P<0.05$ for each). 
Table 4

Correlation of the Hcy and serum lipid parameters

\begin{tabular}{|llllll|}
\hline $\begin{array}{l}\text { Relative } \\
\text { factor }\end{array}$ & $\begin{array}{l}\text { Unstandardized } \\
\text { coefficient }\end{array}$ & $\begin{array}{l}\text { Std. } \\
\text { error }\end{array}$ & $\begin{array}{l}\text { Standardized } \\
\text { coefficient }\end{array}$ & $\boldsymbol{t}$ & $\boldsymbol{P}$ \\
\hline Total & & & & -3.053 & 0.002 \\
\hline LDL-C & -0.465 & 0.152 & -0.075 & -5.097 & 0.000 \\
\hline ApoAI & -2.458 & 0.482 & -0.125 & & \\
\hline Control & & & & -2.768 & 0.006 \\
\hline TG & -0.616 & 0.223 & -0.100 & -2.287 & 0.022 \\
\hline HDL-C & -1.614 & 0.706 & -0.083 & & \\
\hline CAD & & & & -2.404 & 0.016 \\
\hline HDL-C & -1.499 & 0.624 & -0.092 & -2.473 & 0.014 \\
\hline ApoAl & -1.652 & 0.668 & -0.095 & & \\
\hline $\begin{array}{l}\text { TC, total cholesterol; TG, triglyceride; HDL-C, high-density lipoprotein cholesterol; LDL-C, low-density } \\
\text { lipoprotein cholesterol; ApoAl, apolipoprotein Al; ApoB, apolipoprotein B }\end{array}$ & \\
\hline
\end{tabular}

\section{Discussion}

$C A D$ is one of the leading causes of morbidity and mortality worldwide. Despite best efforts, available therapies protect only $30-40 \%$ of individuals at risk ${ }^{[14]}$. Thus, it is important to investigate new predictors of CAD to help protect against and provide a new treatment for CAD. A possible relationship between HHcy and CAD was first suggested by Wilcken and Wilcken in $1976^{[27]}$. Since then, more data from various epidemiological investigations and laboratory studies have demonstrated that an increased concentration of serum Hcy was considered to be an independent risk factor for CVD. A meta-analysis showed that an increase of $5 \mu \mathrm{mol} / \mathrm{L}$ in plasma homocysteine level enhances the risk of CVD by 1.6- to 1.8-fold, which is similar to the risk seen with an increase of $20 \mathrm{mg} / \mathrm{dL}(0.52 \mathrm{mmol} / \mathrm{L})$ in cholesterol concentration ${ }^{[28]}$. However, the mechanism for this risk remains unclear. It is well known that serum lipid levels are the most important risk factors for CAD. In the present study, perhaps due to the effects of lipidlowering drugs, there were no significant increases in the levels of serum TC, TGs, LDL-C, or ApoB in CAD patients, but the serum HDL-C and ApoAl were lower in the CAD patients. We also found that serum Hcy was higher in CAD patients. Therefore, we speculate that HHcy may affect the occurrence of CAD by affecting the blood lipid profile, especially HDL-C and ApoAI.

In the present research, statistical analysis was performed between normal Hcy and HHcy groups according to whether the serum homocysteine concentration was greater than $15 \mu \mathrm{mol} / \mathrm{L}$. The levels of serum TC, LDL-C and ApoB in control subjects with HHcy and the levels of serum HDL-C and ApoAl in CAD subjects with HHcy were significantly lower than those of individuals with normal Hcy $(P$ for all < 
0.05). There were also some invaluable clinical observations that demonstrate the possible link between Hcy and lipid metabolism pathways. Most research findings suggest that Hcy is significantly and negatively correlated with HDL-C and ApoAl in CAD or community-based populations. HDL and ApoAI exert anti-atherogenic effects by transporting cholesterol from cells into peripheral tissues ${ }^{\text {[29], reducing }}$ oxidative stress and suppressing inflammatory pathways ${ }^{[30]}$. Low ApoAI (HDL-C) levels are a risk factor for atherosclerosis. Recent animal and in vitro cell studies have also demonstrated that Hcy suppresses hepatic ApoAl expression via the peroxisome proliferator-activated receptor a (PPARa)-ApoAl pathway [24]. Moreover, Hcy could decrease the transcription of ApoAl by stimulating nuclear factor KB (NF-KB) and ApoAl regulatory protein-1 (ARP-1) ${ }^{[23]}$ and enhancing HDL cholesterol clearance ${ }^{[22]}$. These increased Hcy levels may impair cardiovascular function via the inhibition of ApoAl expression and the impairment of its antioxidant capacity [21]. In the present study, although the subjects enrolled in this study had been treated with statins prior to angiography, it is well known that the effect of statins on blood lipids is mainly reflected by lower TC and LDL-C levels and less on HDL-C and ApoAl levels. The negative correlation between HHcy and HDL-C and ApoAI was confirmed by multivariate regression analysis, which suggests that serum Hcy may promote CAD by disturbing HDL (ApoAl) metabolism.

The interaction between Hcy and serum TC, TGs and LDL-C has been explored in some small sample clinical observation studies. Durdi et al. reported that in 126 myocardial infarction patients, Hcy was positively correlated with LDL-C levels ${ }^{[31]}$. In 300 Indian subjects with proven CAD, Hcy was found to be positively associated with TGs and very low-density lipoprotein cholesterol (VLDL-C) ${ }^{[32]}$. In northern Chinese subjects, the prevalence of HHcy in the combined hyperlipidemia group was reported to be significantly higher than that in the control group, with an OR of $3.339{ }^{[33]}$. There are also two communitybased studies that incorporated large samples in China; Momin M et al. showed that HHcy was independently associated with hypertriglyceridemia ${ }^{[34]}$, and Qin YY et al. showed that HHcy was related to high concentrations of TC, TGs, and LDL-C ${ }^{[35]}$. However, not all prior studies have found correlations between HHcy and lipid profiles ${ }^{[36,37]}$. Importantly, the most recent data, including 18297 US adults from the Very Large Database of Lipids (VLDL-21), indicate that, in unadjusted analysis, levels of LDL-C and non-HDL-C were lower, whereas levels of TGs and VLDL-C were higher in the highest Hcy quartile, but after adjusting for confounders, the associations disappeared ${ }^{[38]}$. In the present study, we found that the levels of serum TC, LDL-C and ApoB in the HHcy group were significantly lower than those in the normal Hcy group in a population treated with lipid-lowering therapy, which appears to suggest that Hcy is associated with lipid parameters in the protection against atherosclerosis in these populations. To explain these contradictions, it must be noted that information on lipid-lowering medication, one of the most important confounders, was uncertain in our study and the VLDL-21 study. Perhaps it is very interesting to explore why individuals with $\mathrm{HHcy}$ who are taking lipid-lowering drugs have healthier blood lipid profiles. However, we lack information about the dose and treatment course of lipid-lowering drugs and the baseline lipid level before treatment. The effect of the interaction between HHcy and lipidlowering drugs on blood lipids needs further research to clarify. Therefore, it is difficult to determine 
whether HHcy promotes atherosclerosis or protects against atherosclerosis by evaluating indicators such as TC, LDL and ApoB that are greatly affected by lipid-lowering drugs.

Nontraditional lipid profiles, including TC/HDL-C, LDL/HDL-C, TG/HDL-C and ApoB/Al, have been found to be independent indicators of vascular risk with greater predictive value than isolated lipid levels. Our current study aimed to evaluate whether HHcy is associated with promoting atherosclerosis or protecting against atherosclerotic lipid profiles by evaluating the lipid ratio. The present study showed that different trends in Hcy affect the ratio of TC/HDL-C and LDL-C/HDL-C between the CAD and controls ( $P$ for interaction $<0.05)$. In the controls, HHcy was associated with a decreased trend in the ratio of TC/HDL-C (normal Hcy: $4.11 \pm 1.03$ vs HHcy: $4.05 \pm 1.11 ; P=0.481$ ) and LDL/HDL-C (normal Hcy: $2.52 \pm 0.80 v s$ HHcy: $2.45 \pm 0.85 ; P=0.303)$. However, CAD patients with HHcy had a significantly higher ratio of TC/HDL-C (normal Hcy: $4.10 \pm 1.32$ vs HHcy: $4.43 \pm 2.60 ; P=0.025$ ) and higher LDL/HDL-C (normal Hcy: $2.48 \pm 1.07 v s \mathrm{HHcy}: 2.65 \pm 1.36 ; P=0.045)$ than normal Hcy patients. The importance of TC/HDL-C and LDL/HDL-C was highlighted in some large studies; Ridker PM showed that the use of either the ratio of TC/HDL-C or that of LDL/HDL-C is superior to the use of TC or LDL-C alone ${ }^{[7]}$. Arsenault BJ et al. observed that among apparently healthy men and women in a cohort representative of a contemporary Western population, the TC/HDL-C ratio was more strongly associated with the risk of future CHD than LDL-C; they also found that at any LDL-C level, individuals with an elevated TC/HDL-C ratio were still at an increased risk of developing CAD ${ }^{[6]}$. Therefore, these findings suggest that $\mathrm{HHcy}$ may play an important role in the atherogenic lipid profile in patients with CAD. In people taking lipid-lowering drugs, the ratio of blood lipids is more suitable for assessing the effect of HHcy on CAD.

\section{Conclusion}

The traditional lipid levels of serum TC, LDL-C, and ApoB in control subjects with HHcy and serum HDL-C and ApoAl in CAD subjects with HHcy were significantly lower than those in individuals with normal Hcy. The effect of HHcy on the ratio of TC/HDL-C and LDL/HDL-C was different between the CAD patients and controls. HHcy was related to the atherogenic lipid ratio in patients with CAD. The ratio of blood lipids is more suitable for assessing the effect of HHcy on CAD.

\section{Abbreviations}

Apo: Apolipoprotein

Apo: ApoAl regulatory protein-1

CVD: Cardiovascular disease

CAD: Coronary artery disease

Hcy: Homocysteine 
HHcy: Hyperhomocysteinemia

HDL-C: High-density lipoprotein cholesterol

MTHFR: Methylene Tetrahydrofolate Reductase

LDL-C: Low-density lipoprotein cholesterol

NF-Kb: Nuclear factor KB

PPARa: Peroxisome proliferator-activated receptor a

TG: Triglycerides

TC: Total cholesterol

VLDL-C: Very low-density lipoprotein cholesterol

\section{Declarations}

\section{Ethics approval and consent to participate}

This study was complied with the Declaration of Helsinki and approved by the Institutional Ethics Committee of People's Hospital of Guangxi Zhuang Autonomous Region. Written informed consent was not obtained from the participants, because of the data retrospectively obtained from electronic medical records.

\section{Consent for publication}

Not applicable.

\section{Availability of data and materials}

The datasets used and/or analyzed during the current study will be available from the corresponding author on reasonable requests after study completion.

\section{Competing interests}

None

\section{Funding}

This study was supported by the National Natural Science Foundation of China (No:81660066), the Natural Science Foundation of Guangxi (No: 2016GXNSFBA380209). The funders had no role in the study design; in the collection, analysis and interpretation of data; in the writing of the manuscript; or in the decision to submit the article for publication. 


\section{Authors' contribution}

DFW participated in the design, performed the statistical analyses, and drafted the manuscript. JLD conceived the study, participated in the design. QCL, FL, ZW, KY collected the data and the samples. All authors read and approved the final manuscript.

\section{Acknowledgements}

None

\section{References}

1. Dele $O$ Abegunde, Colin D Mathers, Taghreed Adam, Monica Ortegon, Kathleen Strong, The burden and costs of chronic diseases in low-income and middle-income countries. 2007; 370(9603):192938.

2. Castelli WP, Garrison RJ, Wilson PW, Abbott RD, Kalousdian S, Kannel WB.Incidence of coronary heart disease and lipoprotein cholesterol levels. The Framingham Study.JAMA. 1986 Nov 28;256(20):2835-8

3. Gordon DJ, Probstfield JL, Garrison RJ, Neaton JD, Castelli WP, Knoke JD, Jacobs DR Jr, Bangdiwala S, Tyroler HA.High-density lipoprotein cholesterol and cardiovascular disease. Four prospective American studies.Circulation. 1989 Jan;79(1):8-15.

4. Bittner V, Johnson BD, Zineh I, Rogers WJ, Vido D, Marroquin OC, BaireyMerz CN, Sopko G. The triglyceride/high-density lipoprotein cholesterol ratio predicts all-cause mortality in women with suspected myocardial ischemia: a report from the Women's ischemia syndrome evaluation (WISE). Am Heart J. 2009;157:548-55》

5. Barzi F, Patel A, Woodward M, Lawes CM, Ohkubo T, Gu D, Lam TH, Ueshima H. A comparison of lipid variables as predictors of cardiovascular disease in the Asia Pacific region. Ann Epidemiol. 2005;15:405-13】

6. Arsenault BJ, Rana JS, Stroes ES, Despres JP, Shah PK, Kastelein JJ, Wareham NJ, Boekholdt SM, Khaw KT. Beyond low-density lipoprotein cholesterol: respective contributions of non-high-density lipoprotein cholesterol levels, triglycerides, and the total cholesterol/high-density lipoprotein cholesterol ratio to coronary heart disease risk in apparently healthy men and women. J Am Coll Cardiol. 2009;55:35-41区

7. Ridker PM, Rifai N, Cook NR, Bradwin G, Buring JE. Non-HDL cholesterol, apolipoproteins A-I and B100, standard lipid measures, lipid ratios, and CRP as risk factors for cardiovascular disease in women. JAMA. 2005;294:326-33】

8. Fernandez ML, Webb D. The LDL to HDL cholesterol ratio as a valuable tool to evaluate coronary heart disease risk. J Am Coll Nutr. 2008;27:1-5.

9. Bhalodkar NC, Blum S, Enas EA. Accuracy of the ratio of triglycerides to high-density lipoprotein cholesterol for predicting low-density lipoprotein cholesterol particle sizes, phenotype B, and particle 
concentrations among Asian Indians. Am J Cardiol. 2006;97:1007-9.

10. Drexel H, Aczel S, Marte T, Benzer W, Langer P, Moll W, Saely CH. Is atherosclerosis in diabetes and impaired fasting glucose driven by elevated LDL cholesterol or by decreased HDL cholesterol? Diabetes Care. 2005;28:101-7》

11. Turak O, Afsar B, Ozcan F, Oksuz F, Mendi MA, Yayla C, Covic A, Bertelsen N, Kanbay M. The role of plasma triglyceride/high-density lipoprotein cholesterol ratio to predict new cardiovascular events in essential hypertensive patients. J Clin Hypertens (Greenwich). 2016;18:772-7.

12. Elshazly MB, Nicholls SJ, Nissen SE, St John J, Martin SS, Jones SR, Quispe R, Stegman B, Kapadia SR, Tuzcu EM, Puri R. Implications of Total to high-density lipoprotein cholesterol ratio discordance with alternative lipid parameters for coronary Atheroma progression and cardiovascular events. Am J Cardiol. 2016;118:647-55.

13. Wang H, Guo X, Chen Y, Li Z, Xu J, Sun Y. Relation of four nontraditional lipid profiles to diabetes in rural Chinese H-type hypertension population. Lipids Health Dis. 2017;16(1):199.

14. Natarajan P, Ray KK, Cannon CP. High-density lipoprotein and coronary heart disease: current and future therapies. J Am Coll Cardiol. 2010, 55: 1283-99.

15. Huo Y, Li J, Qin X, et al. Efficacy of folic acid therapy in primary prevention of stroke among adults with hypertension in China: the CSPPT randomized clinical trial. JAMA. 2015;313(13):1325-35.

16. Antoniades C, Antonopoulos AS, Tousoulis D, Marinou K, Stefanadis C. Homocysteine and coronary atherosclerosis: from folate fortification to the recent clinical trials. Eur Heart J. 2009;30(1):6-15;

17. Wierzbicki AS. Homocysteine and cardiovascular disease: a review of the evidence. Diab Vasc Dis Res. 2007; 2007:143.

18. Xiao Y, Zhang Y, Lv X, Su D, Li D, Xia M, Qiu J, Ling W, Ma J. Relationship between lipid profiles and plasma total homocysteine, cysteine and the risk of coronary artery disease in coronary angiographic subjects. Lipids Health Dis. 2011;10:137;

19. Herrmann W, Obeid R, Hubner U, Jouma M, Geisel J. Homocysteine in relation to C-reactive protein and low-density lipoprotein cholesterol in assessment of cardiovascular risk. Cell Mol Biol (Noisy-legrand). 2004;50(8):895-901;

20. Daly C, Fitzgerald AP, O'Callaghan P, Collins P, Cooney MT, Graham IM. Homocysteine increases the risk associated with hyperlipidaemia. Eur J Cardiovasc Prev Rehabil. 2009;16(2):150-5.

21. Yang N, Yao Z, Miao L, Liu J, Gao X, Xu Y, Wang G.Homocysteine diminishes apolipoprotein A-I function and expression in patients with hypothyroidism: a cross-sectional study. Lipids Health Dis. 2016;15:123.

22. Liao D, Tan H, Hui R, Li Z, Jiang X, Gaubatz J, Yang F, Durante W, Chan L, Schafer Al, Pownall HJ, Yang $X$, Wang $H$. Hyperhomocysteinemia decreases circulating high-density lipoprotein by inhibiting apolipoprotein A-I Protein synthesis and enhancing HDL cholesterol clearance. Circ Res. 2006;99(6):598-606.

23. Wang Y, Liu J, Jiang Y, Zhang H, Leng S, Wang G. Hyperhomocysteinemia is associated with decreased apolipoprotein Al levels in normal healthy people. BMC Cardiovasc Disord. 2016;16:10. 
24. Mikael LG, Genest Jr J, Rozen R. Elevated homocysteine reduces apolipoprotein A-l expression in hyperhomocysteinemic mice and in males with coronary artery disease. Circ Res. 2006;98:564-71.

25. Wu DF, Yin RX, Cao XL, Chen WX, Aung LHH, Wang W, Huang KK, Huang P, Zeng XN, Wu J. Scavenger Receptor Class B Type 1 Gene rs5888 Single Nucleotide Polymorphism and the Risk of Coronary Artery Disease and Ischemic Stroke: A Case-Control Study. Int J Med Sci. 2013; 10(12):1771-1777.

26. Wu DF, Wu YX, Deng JL. Changes in Homocysteine Levels Affect Serum Lipid Response to Atorvastatin in Patients With Acute Coronary Syndrome: A Retrospective Observational Study. Clin Appl Thromb Hemost. 2020;26:1076029620920369.

27. Wilcken DEL, Wilcken B. The pathogenesis of coronary artery disease: a possible role for methionine metabolism. J Clin Invest 1976; 57:1079-82.

28. Boushey CJ, Beresford SA, Omenn GS, Motulsky AG. A quantitative assessment of plasma homocysteine as a risk factor for vascular disease. Probable benefits of increasing folic acid intakes. JAMA 1995; 274: 1049-57

29. Asztalos BF, Horvath KV, Schaefer EJ. High-Density Lipoprotein Particles, Cell-Cholesterol Efflux, and Coronary Heart Disease Risk. Arterioscler Thromb Vasc Biol. 2018;38(9):2007-2015.

30. Didichenko SA, Navdaev AV, Cukier AM, Gille A, Schuetz P, Spycher MO, Thérond P, Chapman MJ, Kontush A, Wright SD. Enhanced HDL Functionality in Small HDL Species Produced Upon Remodeling of HDL by Reconstituted HDL, CSL112: Effects on Cholesterol Efflux, Anti-Inflammatory and Antioxidative Activity. Circ Res. 2016;119(6):751-63.

31. Qujeq D, Omran TS, Hosini L. Correlation between total homocysteine, low-density lipoprotein cholesterol and high-density lipoprotein cholesterol in the serum of patients with myocardial infarction. Clin Biochem. 2001;34(2):97-101.

32. Mahalle N, Kulkarni MV, Garg MK, Naik SS. Vitamin B12 deficiency and hyperhomocysteinemia as correlates of cardiovascular risk factors in Indian subjects with coronary artery disease. $\mathrm{J}$ Cardiol. 2013;61(4):289-94.

33. Huang L, Song XM, Zhu WL, Li Y. Plasma homocysteine and gene polymorphisms associated with the risk of hyperlipidemia in northern Chinese subjects. Biomed Environ Sci. 2008;21(6):514-20.

34. Yadav AS, Bhagwat VR, Rathod IM. Relationship of plasma homocysteine with lipid profile parameters in ischemic heart disease. Indian J Clin Biochem. 2006;21(1):106-10.

35. de Luis DA, Fernandez N, Arranz ML, Aller R, Izaola O, Romero E. Total homocysteine levels relation with chronic complications of diabetes, body composition, and other cardiovascular risk factors in a population of patients with diabetes mellitus type 2. J Diabetes Complications. 2005;19(1):42-6.

36. Momin M, Jia J, Fan F, Li J, Dou J, Chen D, Huo Y, Zhang Y. Relationship between plasma homocysteine level and lipid profiles in a community-based Chinese population. Lipids Health Dis. 2017;16(1):54.

37. Qin YY, Wang P, Qin JQ, Wei AQ, Huang P, Lai ZF, Lin FQ. Prevalence of hyperhomocysteinemia during routine physical examination in Guangxi Province, China and related risk factors. J Clin Lab Anal. 2018;32: e22178. 
38. Lupton JR, Quispe R, Kulkarni K, Martin SS, Jones SR. Serum homocysteine is not independently associated with an atherogenic lipid profile: The Very Large Database of Lipids (VLDL-21) study. Atherosclerosis. 2016; 249:59-64. 